\title{
Zoster Laryngitis with Multiple Cranial Nerve Palsy Progressed as Ascending Involvement
}

\author{
Woosub Hwang, $\mathrm{MD}^{1}$, Da-Eun Jeong, $\mathrm{MD}^{2}$ \\ ${ }^{1}$ Department of Neurology, Daegu Fatima Hospital, Daegu; ${ }^{2}$ Department of Neurology, Yeungnam University College of Medicine, Daegu, Korea
}

Background: Herpes zoster of the head and neck commonly presents with Ramsay Hunt syndrome. However, vesicular eruptions may occur on the pharyngeal or laryngeal area with multiple lower cranial-nerve (CN) palsy.

Case Report: We report on the case of a 54-year-old man with herpes zoster of the pharynx and larynx with multiple CN palsy and persistent hiccups. He initially developed progressive dysphagia, hoarseness, and persistent hiccups (CN IX and X). After admission, Dizziness, hearing impairment, and peripheral facial palsy (CN VII and VIII) were complicated. The results of a polymerase chain reaction test of saliva and vesicular fluid from the ear and throat were strongly positive for varicella zoster virus. The progression of CN palsy was in an ascending sequence.

Conclusion: We suggest that the sequence of $\mathrm{CN}$ palsy may be either ascending or descending, depending on the initial site of involvement.

J Neurocrit Care 2018;11(1):43-46

Key words: Herpes zoster; Cranial nerve diseases; Laryngitis; Hiccup

\author{
Received May 21, 2018 \\ Revised May 24, 2018 \\ Accepted June 4, 2018 \\ Corresponding Author: \\ Da-Eun Jeong, MD \\ Department of Neurology, Yeungnam \\ University College of Medicine, 170 \\ Hyeonchung-ro Nam-gu, Daegu 42415, \\ Korea \\ Tel: +82-53-620-3684 \\ Fax: +82-53-627-1688 \\ E-mail: doctorjung86@gmail.com
}

Copyright $\odot 2018$ The Korean Neurocritical Care Society

\section{INTRODUCTION}

Herpes zoster of the head and neck commonly presents with Ramsay Hunt syndrome (RHS). ${ }^{1-3}$ However, vesicular eruptions may occur on the pharyngeal or laryngeal area with multiple lower cranial-nerve (CN) palsy, ${ }^{1-10}$ meningoencephalitis, and vasculopathy. We herein report a case of RHS with vesicles in the pharynx and larynx, complicated by multiple lower $\mathrm{CN}$ palsy and persistent hiccups in an immunocompetent patient.

\section{CASE REPORT}

A 50-year-old man presented with a 3-day history of progressive dysphagia, hoarseness, fever, and persistent hiccups. He also complained of facial pain on the right side. He had been healthy until three days prior to admission. He had a history of chickenpox in his childhood and recently had had severe emotional distress.

Upon presentation, blood pressure was $130 / 80 \mathrm{mmHg}$ and body temperature was $39.2^{\circ} \mathrm{C}$. Physical examination revealed multiple small vesicles over the cheek, ear, oral

(cc) This is an Open Access article distributed under the terms of the Creative Commons Attribution Non-Commercial License (http://creativecommons.org/licenses/by$\mathrm{nc} / 4.0$ ) which permits unrestricted non-commercial use, distribution, and reproduction in any medium, provided the original work is properly cited. 
cavity, and pharynx on the right side. There was paralysis of the soft palate and reduced gag reflex on the right side. However, he had no meningeal irritating signs, facial palsy, hearing loss, tongue deviation, or weakness of the sternocleidomastoid and trapezius muscles. The result of a Tzanck test of the vesicles was positive. Initial brain magnetic resonance imaging (MRI) showed no abnormality. He was diagnosed with herpes zoster with CN IX and X palsy. We started intravenous acyclovir (30 mg/kg/day) and dexamethasone (20 mg/day) for herpes zoster, and oral valproic acid and topiramate for intractable hiccups.

On the next day, his hoarseness became worse. Because he could not swallow food or fluid due to severe dysphasia, we started tube feeding. Laryngeal fibroscopy disclosed multiple vesicles and ulcers on the larynx, pharynx, epiglottis, and vocal cord palsy at the right side (Fig. 1). Laboratory tests showed a white blood cell count of $8,460 / \mathrm{mm}^{3}$ with $70.7 \%$ neutrophils, $13.7 \%$ lymphocytes, and $8.4 \%$ monocytes. C-reactive protein was significantly increased, with a concentration of $4.6 \mathrm{mg} / \mathrm{dL}$ (range, $<0.06 \mathrm{mg} / \mathrm{dL}$ ), and an erythrocyte sedimentation rate of $31 \mathrm{~mm} / \mathrm{h}$ (range, $<20 \mathrm{~mm} / \mathrm{h}$ ). Other routine laboratory investigations were within normal ranges. Varicella-zoster virus (VZV), immu- noglobulin (Ig) G, and Ig M antibody were positive, 28.30 and 7.32, indicating reactivation of VZV. A serum polymerase chain reaction (PCR) test for $V Z V$ deoxyribonucleic acid (DNA) was weakly positive. However, the results of a PCR test of saliva and vesicular fluid from the ear and throat were strongly positive (Fig. 2). The patient was immunocompetent on laboratory investigations: hematological screening for Human immunodeficiency virus and lymphocyte subpopulations count, serum immunoglobulins, and complement C3 and C4.

Three days after admission, the patient developed dizziness, hearing impairment, and peripheral facial palsy on the right side. Hoarseness and dysphagia were aggravated. Neurological examination showed spontaneous left-beating nystagmus with delayed vestibulo-ocular reflex upon rightward head rotation. A caloric test disclosed a vestibulopathy and an audiogram revealed a mild sensory neural hearing loss in the right ear. On the fourth day, a followup MRI scan with enhanced contrast revealed an enlarged right facial nerve in the area adjacent to the right ear (Fig. 3). Fifth days after admission, dysphagia and hoarseness gradually improved. On the seventh day, facial palsy, dizziness, and hiccups were beginning to improve. After a total
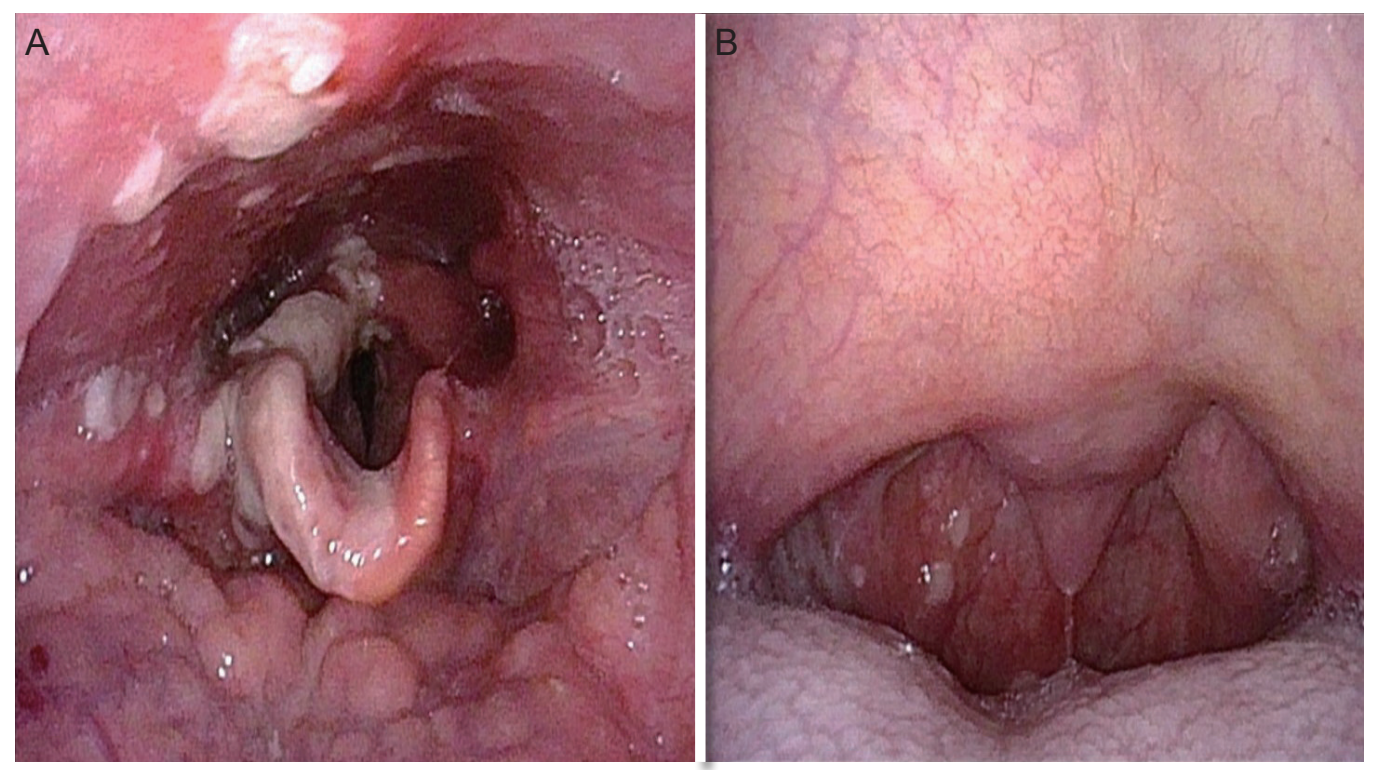

Figure 1. Flexible laryngoscopy (A) showing multiple vesicles and ulcers with erythematous base on the right epiglottis and larynx, as well as right-side vocal cord palsy. (B) showing multiple small vesicles on the right pharynx and impaired right-side pharyngeal wall contraction and slight uvular deviation to the left-side. 


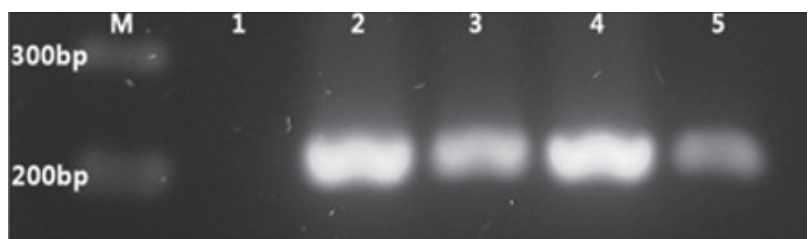

Figure 2. Detection of VZV DNA from saliva and vesicular fluid by RT-PCR test. M, molecular weight marker (100 bp DNA ladder); Lane 1, MRC-5 cell lysate (negative control); Lane 2, VZVinfected Medical Research Council cell strain 5 cell lysate (positive control); Lane 3-5, saliva, vesicular fluid from the ear and throat, respectively. Probes directed to target VZV DNA were of the 202-bp product. VZV, Varicella-zoster virus; DNA, deoxyribonucleic acid; RT-PCR, reverse transcription polymerase chain reaction.

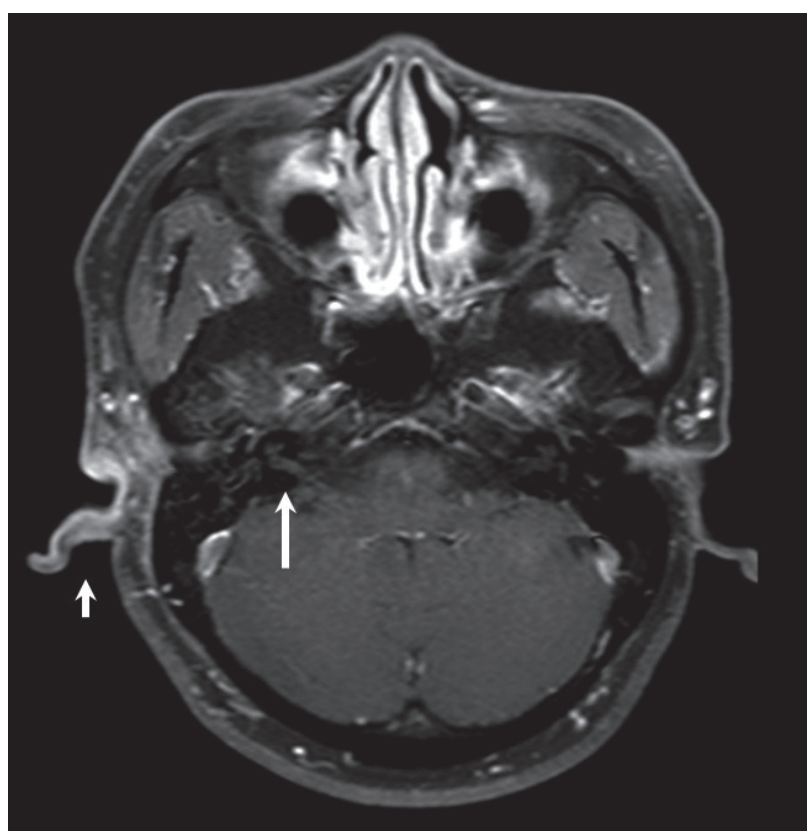

Figure 3. Axial T1-weighted gadolinium-enhanced magnetic resonance imaging showing slight enlargement of the right facial nerve (long arrow) and enlargement and swelling of the right ear and adjacent area (short arrow).

of a 14-day course of acyclovir and corticosteroid treatment, he was discharged in a much-improved state. After 15 months, he had mild facial palsy and hoarseness, but no hearing loss, dizziness, dysphagia, or hiccups.

\section{DISCUSSION}

Reactivation of the VZV is facilitated by diminution of specific cellular immunity to VZV, as happens during the ageing process or in immunosuppressive conditions, following emotional stress or recent viral infection. ${ }^{1,6}$ The virus reactivates in the ganglionic neurons along the entire neuraxis and causes herpes zoster. ${ }^{6}$ However, our patient proved immunocompetent based on laboratory investigations and had no history of immunosuppressive therapy or malignancy, only emotional stress. In the head and neck area, herpes zoster may occur in uncommon patterns, such as vesicular eruptions on the pharyngeal and laryngeal area with lower $\mathrm{CN}$ palsy, intractable hiccups, or facial pain without skin eruption. ${ }^{1-11}$ We present a case of herpes zoster with vesicular eruptions on the pharyngeal and laryngeal area as well as the face, further complicated by intractable hiccups and multiple lower CN palsy, including CN VII, VIII, IX, and X

Case reports of multiple lower $\mathrm{CN}$ involvement due to RHS have rarely been described; zoster laryngitis with cranial polyneuropathy, ${ }^{1-4,6-10}$ and herpes zoster with CN VII, $\mathrm{VIII}$, and $\mathrm{X}$ palsy in an immunocompetent patient. ${ }^{5}$ However, there has been no case report of pharyngeal and laryngeal zoster, similar to our patient, complicated by multiple CN palsy and persistent hiccups in the literature. Only a case of multiple $\mathrm{CN}$ palsy and persistent hiccups without vesicular eruption was reported. ${ }^{11}$

The pathogenic mechanism of multiple CN palsy in herpes zoster has not been clearly elucidated. There have been reports about communication of lower CNs. ${ }^{12-14}$ Recently it was reported that there are various communications among $\mathrm{CN}$ branches around the acoustic meatus and the auricle before reaching the central nervous system; CN X had communications with the CN VII and IX. ${ }^{15}$ We suggest that through these anatomical communications, VZV can spread to neighboring CNs and cause multiple CN palsy.

In our case, he developed dysphagia, hoarseness, and persistent hiccups (CN IX and X) initially, then hearing impairment and facial palsy (CN VII and VIII) after admission. $\mathrm{CN}$ involvement was distinctive as a progressive and ascending sequence, starting from CN IX and X, then spreading to CN VII and VIII. In the previous case reports, the sequence of CNs was variable, ascending, ${ }^{1,2,11}$ descending, ${ }^{4,10}$ 
or not exactly described. ${ }^{3,5}$ So we suggest that the sequence of $\mathrm{CN}$ palsy depends on the initial site of $\mathrm{CN}$ involvement. Then the VZV spreads to adjacent CNs through anatomical communications, either ascending or descending. Patient with herpes zoster with hoarseness and bulbar palsy may develop RHS and vice versa. For this reason, physicians should pay attention to the possibility of the progression of CN palsy for patients with bulbar palsy or facial palsy.

Because cases of laryngeal and pharyngeal herpes zoster with multiple CN palsy and persistent hiccups are uncommon, it is important to rule out other causes of multiple $\mathrm{CN}$ palsy. CN involvement in herpes zoster may progress to adjacent CNs through anatomical communications among CNs. We suggest that the sequence may be either ascending or descending, depending on the initial site of involvement. For this reason, physicians should pay attention to possibility of the progression of $\mathrm{CN}$ palsy.

\section{Conflicts of interest}

The authors have no funding, financial relationships, or conflicts of interest to disclose.

\section{Acknowledgements}

The authors thank Se-Jin Lee for reviewing the manuscript.

\section{REFERENCES}

1. Shinha T, Krishna P. Ramsay Hunt syndrome and zoster laryngitis with multiple cranial nerve involvement. IDCases 2015;2:47-8.

2. Gómez-Torres A, Medinilla Vallejo A, Abrante Jiménez A, Esteban Ortega F. Ramsay-Hunt syndrome presenting laryngeal paralysis. Acta Otorrinolaringol Esp 2013;64:72-4.

3. Van Den Bossche P, Van Den Bossche K, Vanpoucke H. Laryngeal zoster with multiple cranial nerve palsies. Eur Arch Otorhinolaryngol 2008;265:365-7.

4. Choi JH. Two cases of pharyngolaryngeal zoster advanced to multiple cranial neuropathy. Am J Otolaryngo/ 2013;34:36972

5. Morelli N, Mancuso M, Cafforio G, Gallerini S, Pittiglio L, Tonelli S, et al. Ramsay-Hunt syndrome complicated by unilateral multiple cranial nerve palsies. Neurol Sci 2008;29:497-8.

6. Morinaka $S$. Herpes zoster laryngitis with intractable hiccups. Auris Nasus Larynx 2009;36:606-8.

7. Nisa L, Landis BN, Giger R, Leuchter I. Pharyngolaryngeal involvement by varicella-zoster virus. / Voice 2013;27:636-41.

8. Lin $\mathrm{YY}, \mathrm{Kao} \mathrm{CH}$, Wang $\mathrm{CH}$. Varicella zoster virus infection of the pharynx and larynx with multiple cranial neuropathies. Laryngoscope 2011;121:1627-30.

9. Coleman C, Fozo M, Rubin A. Ramsay Hunt syndrome with severe dysphagia. / Voice 2012;26:e27-8.

10. Lee DH, Yoon TM, Lee JK, Joo YE, Lim SC. Herpes zoster laryngitis accompanied by Ramsay Hunt syndrome. / Craniofac Surg 2013;24:e496-8.

11. Yoshida T, Fujisaki N, Nakachi R, Sueyoshi T, Suwazono $S$, Suehara M. Persistent hiccups and vomiting with multiple cranial nerve palsy in a case of zoster sine herpete. Intern Med 2014;53:2373-6.

12. Tanaka S, Mizukami S. Vagal communicating branches between the facial and glossopharyngeal nerves, with references to their occurrence from the embryological point of view. Acta Anat (Basel) 1991;142:25-32

13. Shoja MM, Oyesiku NM, Griessenauer C), Radcliff V, Loukas $M$, Chern J), et al. Anastomoses between lower cranial and upper cervical nerves: a comprehensive review with potential significance during skull base and neck operations, part I: trigeminal, facial, and vestibulocochlear nerves. Clin Anat 2014;27:118-30

14. Shoja MM, Oyesiku NM, Shokouhi G, Griessenauer C), Chern JJ, Rizk EB, et al. A comprehensive review with potential significance during skull base and neck operations, Part II: glossopharyngeal, vagus, accessory, and hypoglossal nerves and cervical spinal nerves 1-4. Clin Anat 2014;27:131-44.

15. Kiyokawa J, Yamaguchi K, Okada R, Maehara T, Akita K. Origin, course and distribution of the nerves to the posterosuperior wall of the external acoustic meatus. Anat Sci Int 2014;89:238-45 